\title{
HARDWARE-IN-THE-LOOP SIMULATION OF HYBRID HYDROMECHANICAL TRANSMISSIONS
}

\author{
L. Viktor Larsson*, Liselott Ericson, Petter Krus \\ Division of Fluid and Mechatronic Systems, Linköping University, 58183 Linköping, Sweden \\ * Corresponding author: Tel.: +46 13281346; E-mail address: viktor.larsson@liu.se
}

\begin{abstract}
Increased demands on fuel-efficient propulsion motivate the use of complex hybrid hydromechanical transmissions in heavy construction machines. These transmissions offer attractive fuel savings but come with an increased level of complexity and dependency on computer-based control. This trend has increased the use of computer-based simulations as a cost-effective alternative to hardware prototyping when developing and testing control strategies. Hardware-In-the-Loop (HWIL) simulations that combine physical and virtual model representations of a system may be considered an attractive compromise that combine the benefits of these two concepts. This paper explores how HWIL simulations may be used to evaluate powertrain control strategies for hybrid hydromechanical transmissions. Factors such as hardware/software partitioning and causality are discussed and applied to a test rig used for HWIL simulations of an example transmission. The results show the benefit of using HWIL simulations in favour of pure offline simulations and prototyping and stress the importance of accurate control with high bandwidth in the HWIL interface.
\end{abstract}

Keywords: Hardware-In-the-Loop Simulations, Hydromechanical Transmissions, Hydraulic Hybrids

\section{INTRODUCTION}

A Hardware-in-the-Loop (HWIL) simulation may be defined as a time-domain simulation of a model that contains both physical (hardware) and virtual (software) representations of a studied system. Within fluid power research in academia, the concept is often used for experimental validation. In an early example (1989), Krus and Palmberg [1] used a servo valve to simulate a load-sensing system connected to a real lorry crane, in a concept referred to as "hybrid simulations". In the automotive industry, HWIL simulation traditionally refers to Controller Hardware-In-the-Loop (CHIL) simulation, which is the real-time testing of control code in a physical control unit (the hardware) that interacts with software system models [2]. This interaction takes place in the signal domain with low power levels. In this paper, HWIL rather refers to Power Hardware-In-the-Loop (PHIL) simulation, which, in contrast to CHIL simulation, has substantial power amplification present in the hardware/software interface [3]. With this feature, HWIL simulation may then be interpreted as an extension to pure offline simulation, where any model component may be replaced with its hardware equivalent, for instance a hydraulic pump or an electric motor. This interpretation of HWIL simulation is illustrated in Figure 1, where the concept is regarded as a middle-way alternative between pure offline simulation and prototype testing of the full system when validating a control strategy.

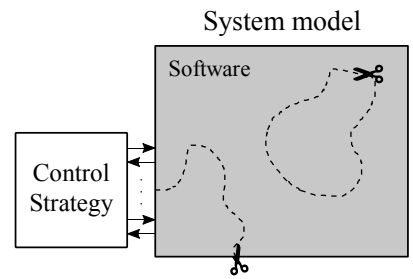

a) Offline simulation

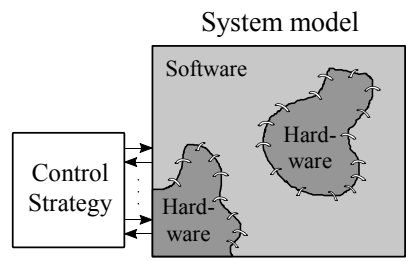

b) HWIL simulation

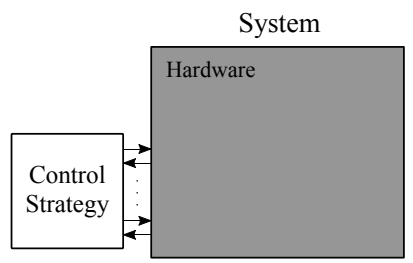

c) Prototype test

Figure 1: Hardware-in-the-loop simulation as a middle way between pure offline simulations and prototype test. 


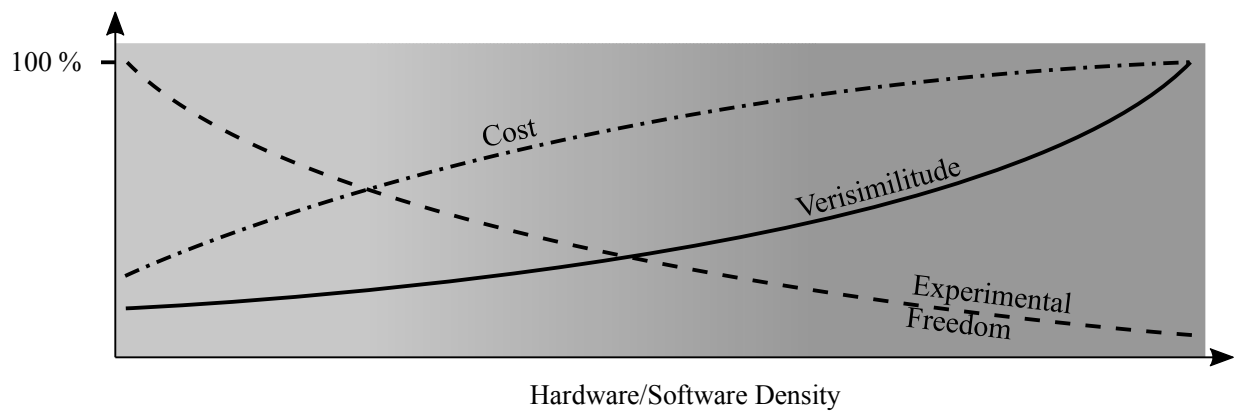

Figure 2: Cost, verisimilitude and experimental freedom.

The choice of HWIL simulation in favour of any of its two extremes may be motivated by comparing their respective pros and cons, as illustrated in Figure 2. Offline simulations offer low cost and high experimental freedom, since, for instance, a model is easy to modify and allows for observation of all the system variables [4]. On the other hand, a full system prototype experiment offers high verisimilitude (truthlikeness). That is, the more hardware that is included in the experiment, the closer to reality it is (should be).

\subsection{Hybrid Hydromechanical Transmissions}

In this paper, a Hydromechanical Trans-mission (HMT) is defined as a transmission that transfers power in both the mechanical and the hydraulic domains, between the engine and the wheels in a vehicle driveline. A hybrid HMT also use hydropneumatic accumulators, that enable energy recuperation and reuse. A general hybrid HMT for a construction machine is shown in Figure 3, and may be represented as a hydraulic circuit with accumulator and variable displacement units connected to a transmission subsystem. The transmission subsystem may contain spur gears and planetary gears to form different transmission architectures, such as input coupled, output coupled or compound power-split [6], depending on the application. For demanding applications, such as heavy wheel loaders, multiple-mode transmissions with clutches are required to fulfil requirements on operating range and cost. A key challenge in the design of these transmissions is the large design space, where simulation-based optimisation has shown to be an important tool [7].

Complex hybrid HMTs also rely heavily on control. As indicated in Figure 3, fast and accurate control of the variable displacement units is required on an actuator level. At a system level, powertrain control manages the engine speed, the system pressure and the operator's

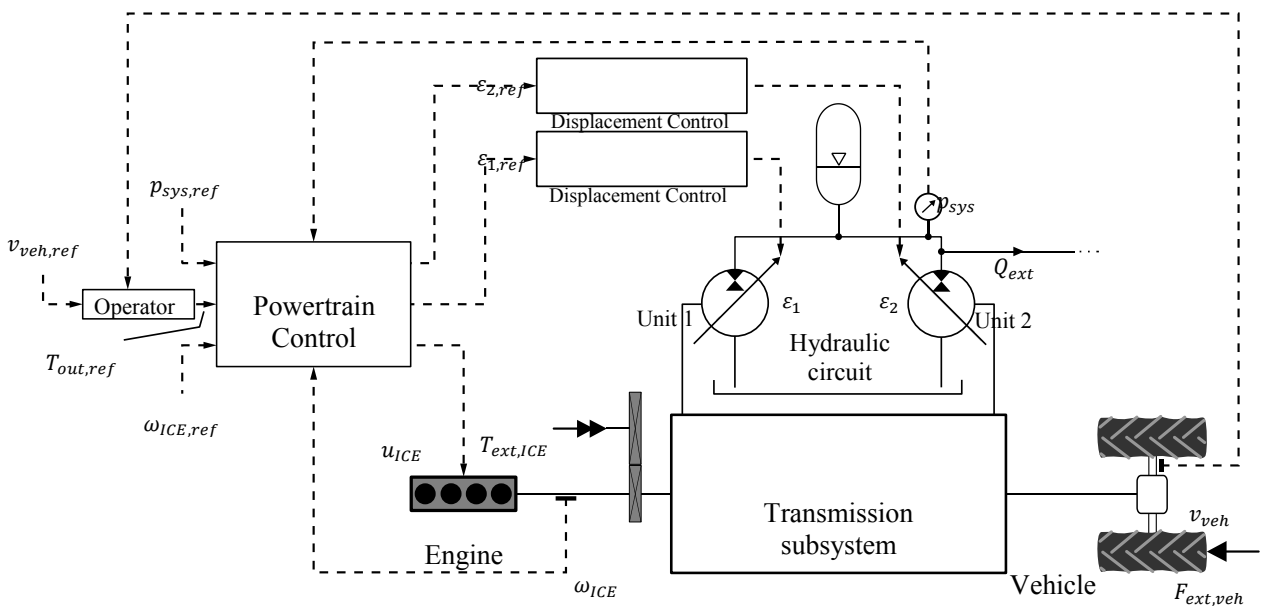

Figure 3: A general hydraulic hybrid drivetrain with its related control aspects and disturbances, from [5]. 
torque request. For hybrid systems, proper energy management of the accumulator and optimal choice of engine operating point are also required to ensure fuel-efficient operation. These topics are out of the scope of this paper but have, for instance, been studied in [8] for on-road vehicles and in [9] for wheel loaders. In Figure 3, it is thus assumed that the system pressure and engine speed reference values are outputs from high- and mid-level strategies that focus on fuel efficiency, while the powertrain control strategy ensures that these values are fulfilled in a stable, accurate manner.

One aspect that distinguishes working machines from e.g. on-road vehicles, is that they have several substantial power consumers in addition to the driveline. These are primarily work functions, such as a loader with boom and bucket, that are actuated with a working hydraulics system. This aspect has big influence on how the machine is designed and controlled [10]. Although the focus in this paper is on the transmission, the presence of additional power consumers is considered as external disturbances in Figure 3. $F_{\text {ext,veh }}$ in Figure 3 indicates an external disturbance force, for instance from a gravel pile.

HWIL simulation has frequently been used in the past for validating and testing control strategies for HMTs. In the early 1990s the concept was applied to hydrostatic transmissions by Jansson et. al. [11] and Lennevi and Palmberg [12]. In the late 1990s, Sannelius [13] used HWIL simulations in the testing of control strategies for the two-motor transmission. A more recent example (2014) is the blended hydraulic hybrid investigated by Sprengel and Ivantysynova [14]. As stressed in [14], HWIL simulations are valuable as alternative to full-scale vehicle tests

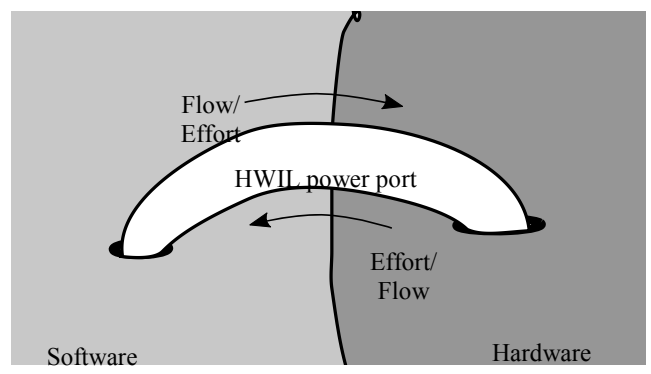

a) Conceptual (modelling) level when evaluating control strategies for novel transmission concepts. Since the transmission does not have to be mounted in a real vehicle, HWIL simulations enable a higher degree of control and repeatability and accurate measurements with high-quality instruments that do not need to be carried on-board the vehicle.

\subsection{Contributions}

This paper explores the application of HWIL simulation as a tool for validation of powertrain control strategies for hybrid HMTs for construction machines. Enablers of HWIL are highlighted and discussed from this perspective, and their implementation in a test rig recently used for this application in [5] is presented. A HWIL simulation is carried out of a reference vehicle for a short cycle and compared to the same cycle in offline simulation.

\section{THE HARDWARE-IN-THE-LOOP INTERFACE}

The key to successful HWIL simulation lies in the hardware/software interface, illustrated in Figure 4. On a conceptual level, the interface may be compared with power ports similar to those used in bond graph modelling [14]. In the HWIL power port, the flow/effort variable is then determined by the software while the effort/flow variable is determined by the hardware. HWIL simulation thus imply a bidirectional hardware/ software communication, which, at the implementation level, is realised with control [2]:

"... a $H(W) I L$ simulator is in essence a control system whose virtual components command its hardware to "track" a hypothetical reference "system"."

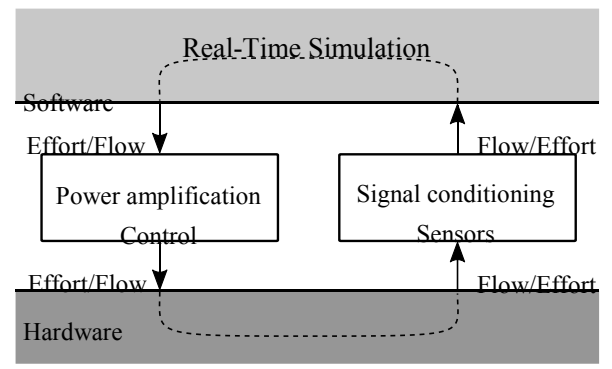

b) Implementation level, adapted from $[1,2]$.

Figure 4: Different levels of the HWIL interface. 
The dependence on real-time control introduces a number of key enablers of HWIL simulation, as listed by Fathy et. al. [2]. For instance, high bandwidth and accuracy of the control is required to maximise the reliability of the results. Consequently, high focus in previous research has been on control aspects of HWIL simulation, e.g. $[11,13,15]$. Another important enabler is the hardware/software integration, which may be divided into partitioning and connection causality [2].

\subsection{Partitioning}

Partitioning relates to the question of how much, and which parts of the studied system that should be represented as hardware or software. The answer to this question is usually a result of the combination of the scope of the investigation and what is feasible (recall Figure 2). In the context of transmission development, Sannelius [13] distinguishes between component focus, where a system is simulated around a new (hardware) component, and system focus, where the new component is simulated in an existing (hardware) system.

If a specific HMT concept is investigated, it is convenient to represent the engine and vehicle as software and the transmission as hardware [14]. For instance, in [11], where a hydrostatic transmission is in focus, the engine is represented as a servo valve-controlled pump/motor while the vehicle is represented as an equivalent inertia (flywheel) with simulated load torque.

When aspects such as emissions and fuel efficiency are studied, the engine is usually represented as hardware connected to a dynamometer which emulates a vehicle, then referred to as Engine-in-the-Loop [16]. With so called rolling road or chassis dynamometers, the complete vehicle is present as hardware, while its surroundings are emulated [17].

The partitioning also determines in which power domain the HWIL interface is inserted, which in turn affects the control implementation. For fluid power systems and hydromechanical transmissions, hydraulic (pressure/flow) or mechanic rotational (torque/angular velocity) interfaces are most often used [13].

\subsection{Connection Causality}

Connection causality relates to the question whether the interface's flow variable (flow/speed) should be determined by the hardware, while the effort variable is determined by the software (pressure/torque), or vice versa. The answer to this question is highly influenced by the behaviour of the hardware and the control performance of the HWIL interface. For instance, the slow variation in pressure caused by the accumulators used in hybrid hydrostatic transmissions motivated a switch from torque to speed control of the output shaft during HWIL simulation of the blended hybrid in [14].

Another example of causality-related difficulties is inertia simulation. If the software controls the speed of a transmission output shaft, vehicle inertia simulation can be carried out via integration of the measured shaft torque. On the other hand, if the software controls the shaft torque, inertia simulation is carried out based on angular acceleration, which often needs to be estimated as the speed time derivative [15]. Another alternative is to represent vehicle inertia as a flywheel and add air drag and other losses via torque control. This is often carried out in rolling road dynamometers, where a set of flywheels can be combined to simulate different vehicle sizes [17]. In a combined approach, Jansson et. al. [11] found that the inertia of the flywheel could be increased around $240 \%$ through estimation of the acceleration, and that higher increases were limited by the stability of the control loop.

\subsection{Fixed-Step Integration}

Fixed-step integration is another key enabler of HWIL simulation. As the interaction between the software and hardware representations of the model takes place in real time, the solver for the software simulation models must be able to finish each integration step within the equivalent step taken by the real-time computer [2]. In this sense, the use of the Transmission Line element Method (TLM) for software system representation is highly motivated. TLM is based on the theory of bilateral delay lines which introduces physically motivated time delays to model physical components [18]. This enables the use of distributed equation solvers without introducing numerical errors and consequently, fast numerically stable simulations with high accuracy may be achieved in real time [19]. In addition, if TLM is used for offline simulation of the full system as well, the same model can be reused during the HWIL simulation without 
making reductions in model fidelity, which facilitates the control strategy development process [7].

In this paper, the Hopsan software is used for all software models. Hopsan is a system simulation tool developed at Linköping University [20]. It uses a fixed-step implementation of TLM and has, for instance, been used for simulation of hydrostatic transmissions and other HMTs in the past, se e.g. $[13,21]$.

\section{TEST RIG}

The test rig is shown in Figure 5 and Figure 6. The basic idea of the setup is to enable HWIL simulation of the generic hybrid hydromechanical transmission shown in Figure 3. In relation to the previously discussed topics, the following implementations are made.

\subsection{Partitioning}

The hardware part of the simulation is the hydraulic circuit, while engine, transmission subsystem and vehicle are represented as software. This partitioning can be motivated by the complex nature of accumulators and hydrostatic unit losses, which are difficult to model mathematically. In addition, testing of different transmission concepts (series hybrid, input/output coupled power-split, multiple-mode etc.) is facilitated via modification of the software model. The hydraulic circuit contains two Bosch A11VO four-quadrant in-line axial piston units with $110 \mathrm{~cm}^{3} / \mathrm{rev}$ displacement. These are equipped with electro-hydraulic displacement actuators that are controlled with external feedback, see $[22,23]$. They are connected in open circuit with two 20 -L piston accumulators connected in parallel on the high-pressure side. A servo-valve is connected to the high-pressure side to simulate the effect of flow disturbances, for instance from a working hydraulics system.

\subsection{Connection causality}

The shaft torques of the hydraulic units are measured in the test rig and sent to the software model, which calculates the corresponding shaft speeds. These values are sent to the rig controller, which controls the actual shaft speeds in closed loop with a servo valve-controlled pump/motor connected to each hydrostatic transmission unit. This causality (measure torque $\rightarrow$ control speed) is motivated by the fact that the hydrostatic units act as torque sources due to the impressed system pressure [14]. No flywheels are used on the shafts, since all vehicle and engine inertial effects are present in the model. This enables fast

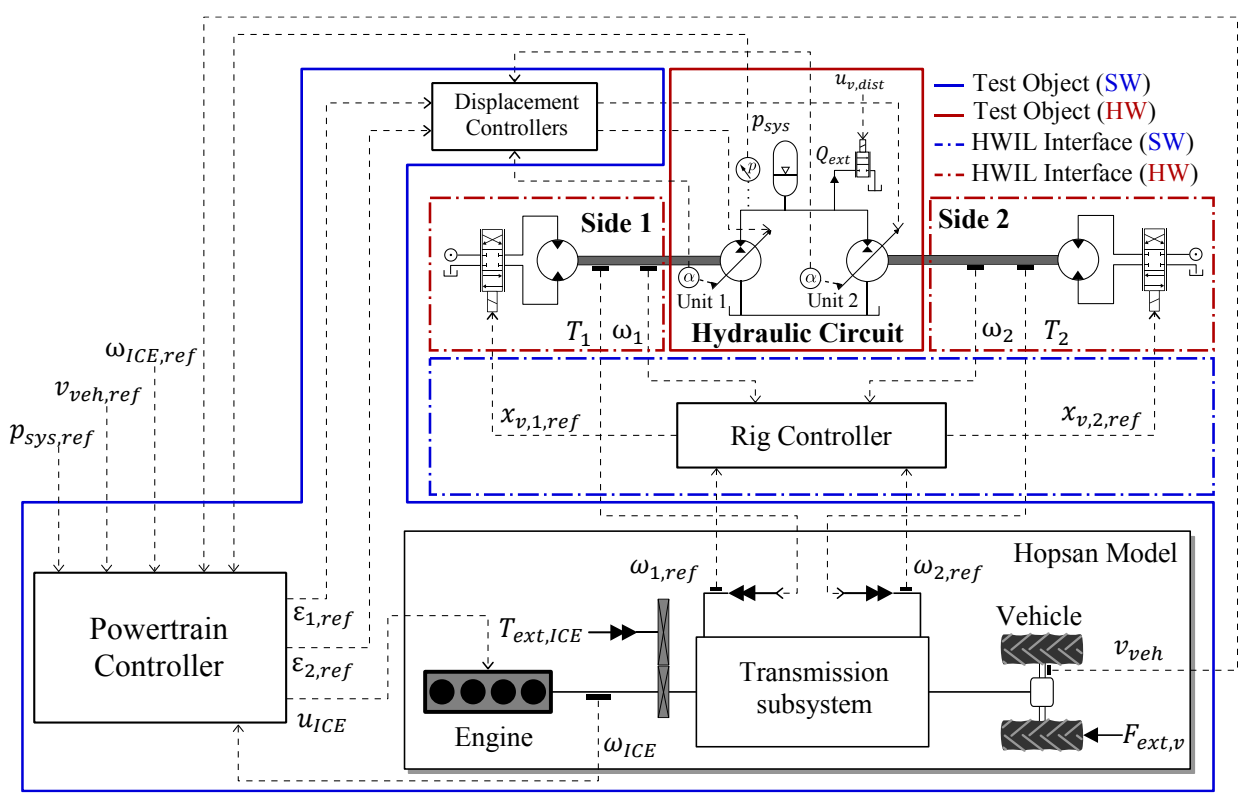

Figure 5: Working principle of the test rig used in the study. SW = Software, HW = Hardware. 


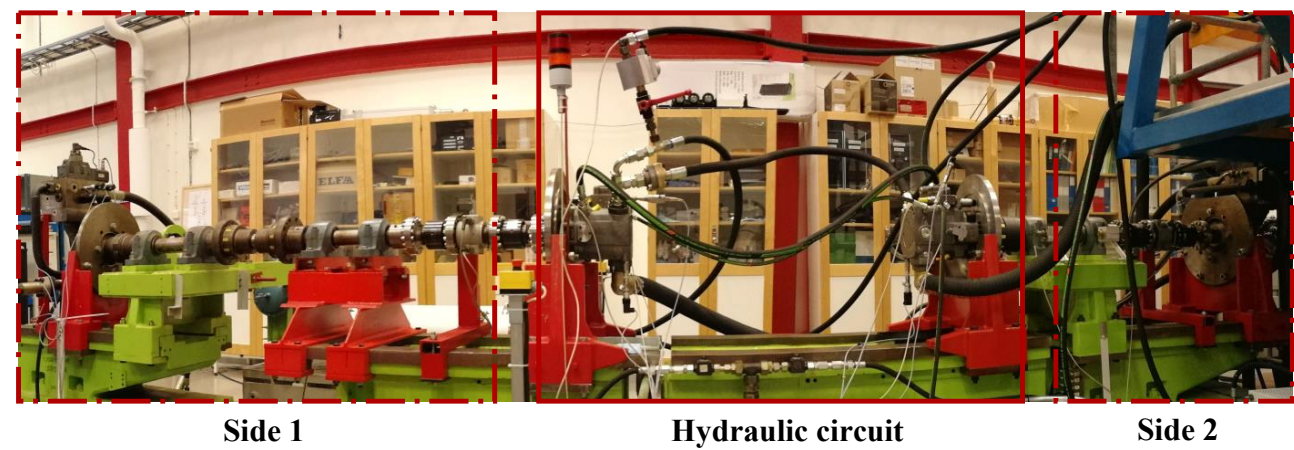

Figure 6: HWIL Simulation test rig main view.

response of the rig shaft speed controllers, although the control becomes sensitive to torque disturbances. The control strategy used in the rig is described in more detail in appendix D in [5], and uses a feedback controller combined with feedforward and disturbance rejection based on estimation of the torque of each transmission pump/motor unit.

\subsection{Real-time and fixed-step integration}

The software model is implemented as a Hopsan model executed on a National Instruments PXI computer that runs in real time with a sampling frequency of $1 \mathrm{kHz}$. This computer also handles the control of the HWIL interface. The full Hopsan offline simulation model is re-used by replacing the hydrostatic circuit with torque sources and adding communication ports. Communication with the PXI computer for software model/controller updates and during HWIL simulation in real time is carried out on a LabVIEW-based Graphical User Interface (GUI) called Viking [24]. See [25] for details on the implementation of the real-time Hopsan simulation and [22] for further details on the data acquisition system hardware.

\section{VEHICLE SIMULATION EXAMPLE}

To illustrate the working principle of the test rig, a reference vehicle equivalent to a compact wheel loader was simulated for a short cycle. The vehicle parameters are provided in Table 1 . The vehicle was tested with an input-coupled powersplit transmission, which means that the transmission's input shaft is directly connected to the hydraulic circuit, while the output shaft is connected to both the hydraulic circuit and the input shaft via a planetary gear train [6].
Table 1: Parameters of the reference vehicle

\begin{tabular}{ll}
\hline Parameter & Value \\
\hline Vehicle mass & $5500 \mathrm{~kg}$ \\
Maximum speed & $30 \mathrm{~km} / \mathrm{h}$ \\
Maximum tractive force & $50 \mathrm{kN}$ \\
Max engine power & $52.7 \mathrm{~kW}$ \\
\hline
\end{tabular}

The reference vehicle was simulated for the same cycle in both HWIL simulation and offline simulation. Figure 7 shows screen shots of the Hopsan models used for these simulations. The offline model represents the complete transmission with hydraulic circuit, diesel engine and vehicle. For the HWIL simulation, the same model was re-used, but the hydraulic circuit was replaced by torque sources fed with the torques measured in the rig. Derivation of the component models used in the Hopsan system models is provided in appendix A in [5].

The powertrain control strategy tested in the simulations has been developed in previous work by the authors $[26,27]$ and is based on decoupled control. In short, this means that a decoupling strategy is used to transform the Multiple-Input Multiple-Output (MIMO) control problem to three Single-Input Single-Output (SISO) loops (system pressure, vehicle speed and engine speed), each controlled with a decoupled control signal. The decoupled control signal for the pressure is the desired net accumulator flow, for the vehicle speed the net transmission output torque and for the engine speed the net engine torque. In the simulations shown, proportional SISO controllers were used, and the tests were carried out by varying of the reference signals (pressure, vehicle speed and engine speed). 


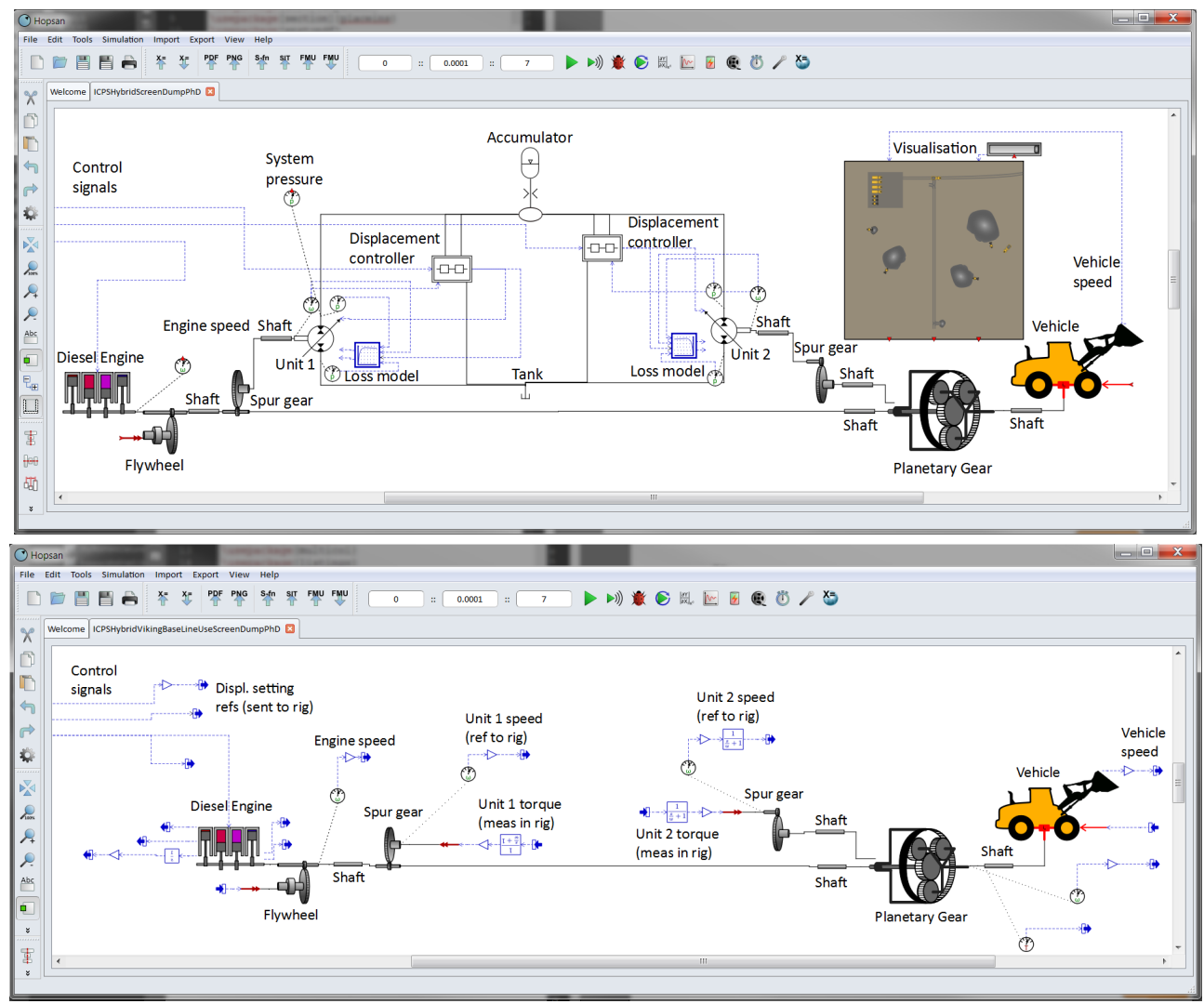

Figure 7: Hopsan models used for the offline (top) and HWIL simulations (bottom). For the HWIL model, the offline model was reused by replacing the hydraulic circuit with torque sources that read filtered signals from the measured rig torques.

\subsection{Results and Discussion}

The simulated cycle for HWIL and offline simulation of the reference vehicle is shown in Figure 8. The cycle aims at simulating a case in which all required vehicle power is provided by the accumulator. As the vehicle is accelerated (13 seconds), the accumulator pressure is lowered, and when it brakes (5-6 seconds), the pressure is increased. The engine speed is controlled at a constant $1800 \mathrm{rpm}$ and is not shown here. The injected engine fuel $\left(u_{I C E}\right)$ is maintained at low levels to overcome engine losses while the accumulator is used to power the vehicle.

The red curves in the two upper graphs indicate the decoupled control signals. For the pressure, the controller demands positive accumulator flow $\left(Q_{a c c, r e f}\right)$ to increase the pressure and negative flow to decrease it. Similarly, the output speed controller may be interpreted as an operator who demands positive output torque ( $\left.T_{\text {out }, \text { ref }}\right)$ to accelerate and negative torque do decelerate. At the lowest control signal level, however, the hydraulic unit displacement settings $\left(\varepsilon_{1}, \varepsilon_{2}\right)$ and the injected engine fuel vary depending on the kinematic relationships of the transmission subsystem.

In the bottom graph in Figure 8, the power flows of the different components during the HWIL simulation are plotted. These have been calculated by multiplying the torque and shaft speeds at the different transmission shafts. $P_{1}$ and $P_{2}$ marks the hydrostatic unit powers, where $P_{1}, P_{2}>0$ indicates pumping mode. The accumulator power has been calculated as $P_{a c c}=$ $P_{1}+P_{2}$. The same graph also includes the consumed fuel of the engine. It may be observed 
that the consumption is lowered when the vehicle energy is recuperated at 5.5 seconds. It should be noted though, that the shown simulation is primarily an example of a cycle, and any estimations in terms of fuel efficiency improvements require comparisons with state-ofthe-art systems and more appropriate energy management strategies.

Benefits with HWIL simulation may be illustrated via a comparison with the offline simulation results. The difference between the two simulations is small in large portions of the cycle. Given that the strategy was developed and validated in offline simulations, the small difference indicates that the strategy would work appropriately in a final implementation.

Between 5 and 6 seconds, however, a significant difference between the offline and HWIL simulated values of $T_{\text {out,ref }}$ may be observed. The reason for this difference is that the friction loss model for the hydraulic units used in the offline simulation is insufficiently accurate in the given operating point. For an input-coupled power-split transmission, the output torque is proportional to the torque of unit 2 , which in turn is controlled by $\varepsilon_{2}$ due to the slowly varying system pressure. The difference in friction losses in the HWIL simulations therefore causes a saturation in $\varepsilon_{2}$ which is not present in the offline simulation. Consequently, output torque is also limited which results in a significant increase in desired output torque at this point (the operator floors the brake pedal). In a real vehicle, this phenomenon is non-desirable, and could be avoided via manipulation of the reference signals (e.g. higher pressure), application of friction brakes or a different transmission design. Particularly the latter action may be taken with relatively low cost compared to if this problem had been discovered in a prototype transmission.

Although the HWIL simulation indeed increases the system knowledge, it is important to note that its fidelity relies heavily on accurate control in the HWIL interface. Figure 9 shows the rig control performance during the simulated cycle. During transients, the rig speed control is sensitive to torque disturbances due to the low inertia of the shafts. For instance, at 5-6 seconds, at which significant differences between offline and HWIL simulations were discovered, the rig control error is around $12 \%$. The previously drawn conclusion concerning the friction loss models could therefore be questioned, and

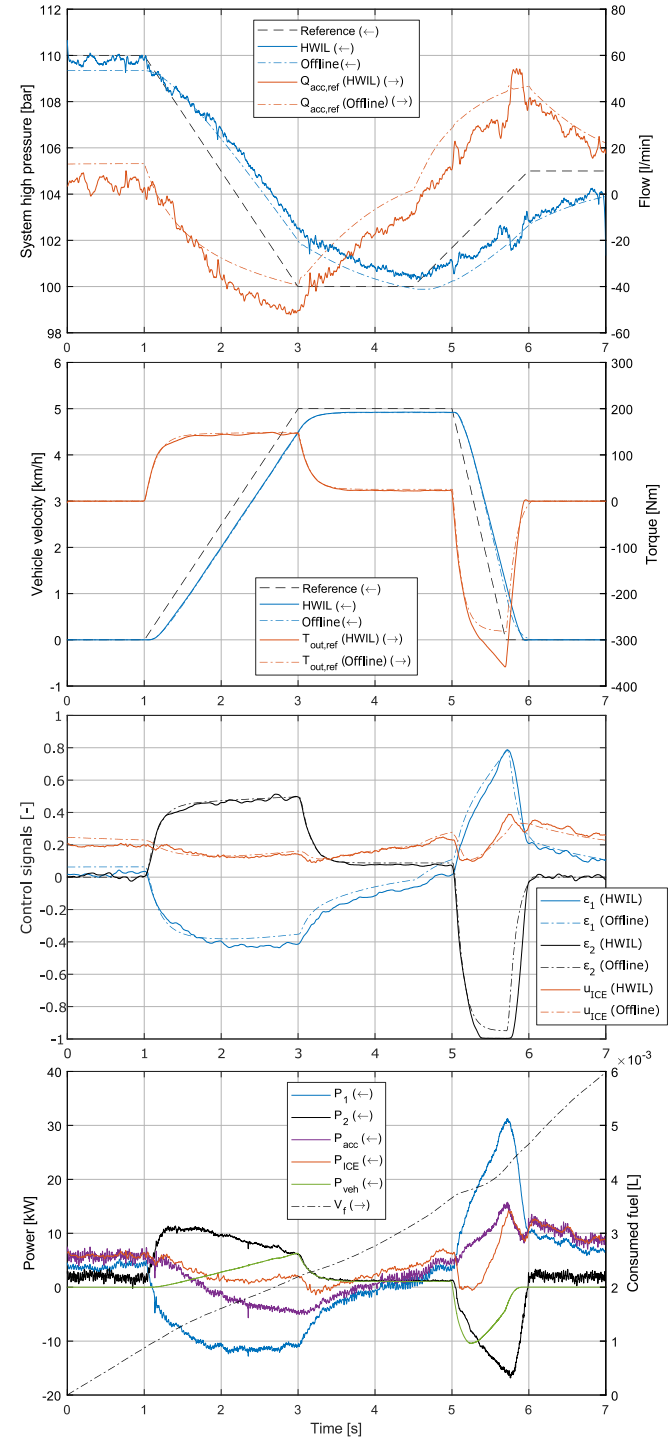

Figure 8: HWIL simulation of the reference vehicle for a short drive cycle. A time step of $0.1 \mathrm{~ms}$ was used in the Hopsan models. Offline denotes results from the offline simulation of the full system.

improvements of the HWIL interface are motivated. The difference in unit shaft torques between the offline and HWIL simulation (Figure 9) at 5-6 seconds does, however, indicate improper loss modelling as a cause of the difference between the offline and HWIL simulations. 

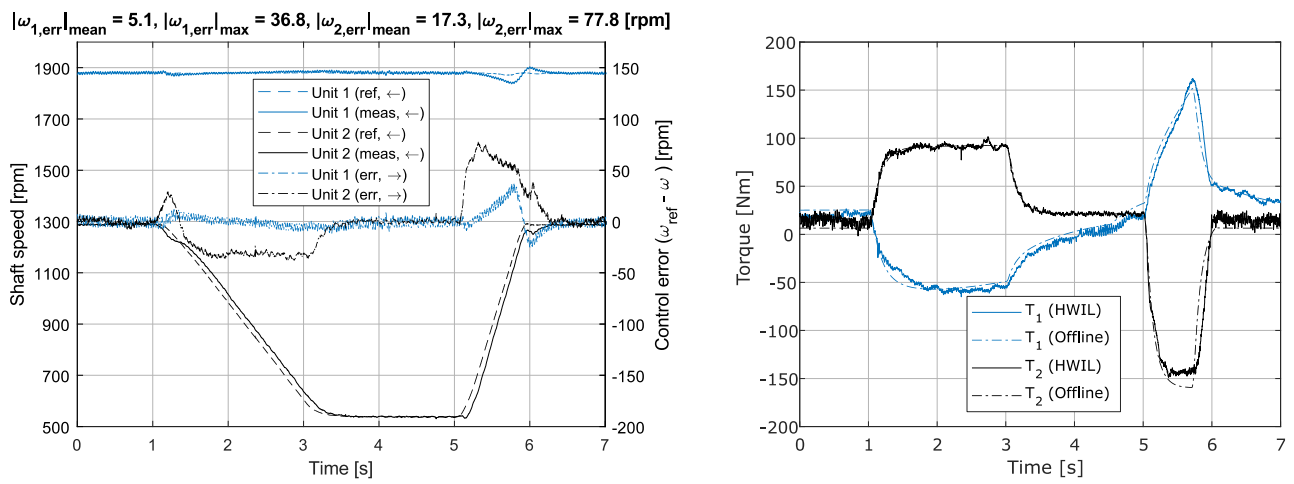

Figure 9: Rig reference tracking (left) and hydraulic unit shaft torques (right) for the drive cycle.

\section{CONCLUSION AND OUTLOOK}

Hardware-in-the-loop simulation is an attractive alternative to offline simulations and prototype testing when evaluating powertrain control strategies for hybrid hydromechanical transmissions in construction machines. By introducing the hydraulic circuit as hardware, the verisimilitude of the simulation increases, while different transmission architectures may be evaluated in a simple manner by changing the software model. This increase in verisimilitude does, however, rely on fast, accurate control in the hardware-in-the-loop simulation interface, and the rig control error should always be considered in model validity assessments. For the presented setup, enhanced control strategies and update of the rig hardware could therefore be reasonable topics for future work. Studies may also be extended to include hardware or software representations of working hydraulics and focus on energy management strategies for construction machines.

\section{NOMENCLATURE}

$\varepsilon_{1 / 2} \quad$ Relative displacement of unit $1 / 2$

$F_{\text {ext,veh }}$ External vehicle disturbance force

$\omega_{1 / 2} \quad$ Shaft speed of unit $1 / 2$

$\omega_{I C E} \quad$ Engine shaft speed

$p_{\text {sys }} \quad$ Transmission high pressure

$Q_{\text {ext }} \quad$ External disturbance flow

$Q_{\text {acc,ref }}$ Desired accumulator net flow

$T_{1 / 2} \quad$ Shaft torque of unit $1 / 2$

$T_{\text {ext,ICE }}$ External engine disturbance torque

$T_{\text {out }, \text { ref }}$ Desired transmission output torque

$u_{I C E} \quad$ Normalised engine injected fuel $\begin{array}{ll}u_{v, \text { dist }} & \text { Disturbance valve signal } \\ v_{v e h} & \text { Vehicle velocity }\end{array}$

$x_{v 1 / 2} \quad$ Servo valve displacement of rig side $1 / 2$

CHIL Controller-Hardware-In-the-Loop

GUI Graphical User Interface

HMT Hydromechanical Transmission

HWIL Hardware-In-the-Loop

ICE Internal Combustion Engine

PHIL Power-Hardware-In-the-Loop

ref Reference value

TLM the Transmission Line element Method

\section{REFERENCES}

[1] Krus P, Palmberg J-O (1989) Integration of Simulation and Measurement in Time and Frequency Domains. The $2^{\text {nd }}$ Int Conf on Fluid Power and Control 512-519

[2] Fathy H, Filipi Z, Hagena J, Stein J (2006) Review of Hardware-in-the-Loop Simulation and Its Prospects in the Automotive Area. Modeling and Simulation for Military Applications 6228

[3] Steurer M, Edrington C, Sloderbeck M, Ren W, Langston J (2010) A Megawatt-Scale Power Hardware-in-the-Loop Simulation Setup for Motor Drives. IEEE Transactions on Industrial Electronics $57.41254-1260$

[4] Fritzson P (2003) Principles of Object Oriented Modeling and Simulation with Modelica 3.3: a cyber-physical approach. Wiley-IEEE Press

[5] Larsson L V (2019) Control of Hybrid Hydromechanical Transmissions. PhD thesis, Linköping University, Linköping, Sweden

[6] Kress J H (1984) Hydrostatic Power-Splitting Transmissions for Wheeled Vehicles - 
Classification and Theory of Operation. SAE Technical Paper 680549

[7] Uebel K (2017) Conceptual Design of Complex Hydromechanical Transmissions. $\mathrm{PhD}$ thesis, Linköping University, Linköping, Sweden

[8] Karbaschian M A, Söffker Dirk (1996) Review and Comparisons of Power Management Approaches for Hybrid Vehicles with Focus on Hydraulic Drives. Energies 7.6 3512-3536

[9] Wang F, Mohd Zulkefli M A, Sun Z, Stelson K A (2015) Energy management strategy for a for a power-split hydraulic hybrid wheel loader. Proceedings of the Institution of Mechanical Engineers, Part D: Journal of Automobile Engineering

[10] Uebel K, Raduenz H, Krus P, De Negri V J (2018) Design Optimisation Strategies for a Hydraulic Hybrid Wheel Loader. ASME/Bath 2018 Symposium on Fluid Power and Motion Control

[11] Jansson A, Lennevi J, Palmberg J-O (1993) Modelling, simulation and control of a load simulator for hydrostatic transmissions. $3^{\text {rd }}$ Int Scandinavian Conf on Fluid Power

[12] Lennevi J, Palmberg J-O (1995) Application and Implementation of LQ Design Method for the Velocity Control of Hydrostatic Transmissions. Proceedings of the Institution of Mechanical Engineers, Part I

[13] Sannelius M (1999) On Complex Hydrostatic Transmissions, Design of a Two-Motor Concept using Computer Aided Development Tools. PhD thesis, Linköping University, Linköping, Sweden

[14] Sprengel M, Ivantysynova, M (2014) Hardware-in-the-Loop testing of a Novel Blended Hydraulic Hybrid Transmission. $8^{\text {th }}$ FPNI PhD Symposium on Fluid Power

[15] Du Z, Li P, Cheong K L, Chase T R (2014) Design and Experimental Validation of a Virtual Vehicle Control Concept for Testing Hybrid Vehicles using a Hydrostatic Dynamometer. The ASME 2014 Dynamic Systems and Control Conf

[16] Filipi Z, Fathy H, Hagena J, Knafl A, Ahlawat R, Liu J, Jung D, Assanis D, Peng H, Stein J (2006) Engine-in-the-Loop Testing for Evaluating Hybrid Propulsion Concepts and Transient Emissions - HMMWV Case Study. SAE 2006 World Congress \& Exhibition

[17] Martyr A J, Plint M A (2007) Engine Testing: Theory and Practice. Elsevier Ltd.
[18] Auslander D M (1968) Distributed System Simulation With Bilateral Delay Models. Journal of Basic Engineering 195-200

[19] Krus P (2009) Robust Modelling using BiLateral Delay Lines for Real Time and Faster Than Real Time System Simulation. ASME 2009 International Design Engineering Technical Conferences \& Computers and Information in Engineering Conference

[20] Linköping University, Division of Fluid and Mechatronic Systems. Hopsan https://liu.se/en/research/hopsan. Last visited: 2020-01-23

[21] Lennevi J (1995) Hydrostatic Transmission Control. PhD thesis, Linköping University, Linköping, Sweden

[22] Larsson L V, Krus P (2016) Modelling of the Swash Plate Control Actuator in an In-Line Axial-Piston Unit for a Hardware-in-the-Loop Simulation Test Rig. The $9^{\text {th }}$ FPNI PhD Symposium on Fluid Power

[23] Larsson L V, Krus P (2017) Displacement Control Strategies of an In-Line Axial-Piston Unit. The $15^{\text {th }}$ Scandinavian International Conference on Fluid Power (SICFP'17)

[24] Prevas (2010) Viking GUI - User Manual

[25] Braun R, Krus P (2012) Multi-threaded realtime simulations of fluid power systems using transmission line elements. The $8^{\text {th }}$ International Fluid Power Conference

[26] Larsson L V, Krus P (2018) A General Approach to Low-Level Control of Heavy Complex Hybrid Hydromechanical Transmissions. ASME/Bath 2018 Symposium on Fluid Power and Motion Control

[27] Larsson L V, Ericson L, Uebel K, Krus P (2019) Low-Level Control of Hybrid Hydromechanical Transmissions for Heavy Mobile Working Machines. Energies 12.9 